\title{
Kalman Filtering for NLOS Mitigation and Target Tracking in Indoor Wireless Environment
}

\author{
Chin-Der Wann \\ National Sun Yat-Sen University \\ Taiwan
}

\section{Introduction}

Kalman filter and its nonlinear extension, extended Kalman filter provide a feasible solution to mitigating non-line of sight (NLOS) propagation effects, and therefore improving accuracy of mobile target tracking in indoor wireless environments. Most wireless communication systems for indoor positioning and tracking may suffer from different error sources, including process errors, measurement errors, the NLOS propagation effects and dense multipath arrivals. The errors sources, if not properly eliminated or mitigated, generally yield severe degradation of accuracy in ranging, positioning and tracking. Among the factors that cause performance degradation, the NLOS effect is considered the major error source in indoor location systems using one or more types of measured location metrics.

Accurate indoor positioning and tracking play an important role in home safety, public services, and other commercial or military applications (Pahlavan et al., 1998). In recent years, indoor localization has drawn increasing interests from academia and industry. There is an increasing demand of indoor localization systems for tracking persons with special needs, such as the elders and children who may be away from visual supervision. Other applications need the solutions to tracing mobile devices or movable objects in the covered areas of sensor networks, or localizing accurately in-demand portable equipments in hospitals and laboratories. In public safety and military operations, the tracking systems can be used in navigating and coordinating police officers, fire-fighters or soldiers to complete cooperative missions inside buildings.

Various positioning techniques have been developed in the past few years. Handset-based positioning methods generally require that a modified handheld device calculate its own position by using a fully or partially equipped global positioning system (GPS) receiver. The method is, however, unfortunately not suitable for many indoor localization applications. Network-based methods have their advantages in wireless location and indoor positioning. The methods can be used for location estimation in situations where GPS solutions are not applicable. In the network-based wireless location, various schemes using received signal strength indication (RSSI) have also been extensively investigated in the past two decades. In location systems using the RSSI, location estimation is usually obtained from or augmented with the location fingerprinting scheme. Though no complex measurement equipments are involved, the build-up of a "radio map" of RSSI may be time-consuming.

Source: Kalman Filter, Book edited by: Vedran Kordić,

ISBN 978-953-307-094-0, pp. 390, May 2010, INTECH, Croatia, downloaded from SCIYO.COM 
The RSSI data may vary from time to time when the layout or environment becomes different from that when the RSSI data are collected. Due to the limitations of spectral natures of transmitted signals in the location systems, accuracy and precision become a major challenge when applications other than "tour-guiding'" services are targeted.

To tackle the problems of mobile positioning and tracking in indoor wireless NLOS environments, a variety of techniques and systems have been studied in recent years in the hope of attaining better location accuracy. Time of arrival (TOA) and time difference of arrival (TDOA) are two typical time-related parameters usually used in pinpointing the location of a mobile station. In addition, techniques using angle of arrival (AOA) have also been studied by many researchers. When line of sight (LOS) transmission exists between a transmitter and a receiver, the signal arrival time or signal arrival angle may be correctly obtained if the SNR is high and the multi-paths from the propagation channels are resolved properly. In situations where NLOS propagation exists, suitable NLOS mitigation techniques are needed for improving the accuracy of ranging and localization.

For applying the TOA and TDOA parameters in locating mobile stations or targets, the true range between a transmitter and a receiver in the wireless environment is correctly calculated only when the direct path of signal propagation is present, which may not always be possible, especially in indoor environments. In most cases, errors caused by the NLOS effects cannot be ignored in wireless location systems where high accuracy is demanded.

Several NLOS mitigation techniques for mobile positioning systems have been presented in the past few years (Le et al., 2003; Najar \& Vidal, 2003; Wylie \& Holtzmann, 1996). In (Wylie \& Holtzmann, 1996), a simple binary hypothesis testing was used for NLOS identification by exploiting the known statistics of the receiver measurement noise. To mitigate the NLOS effects, polynomial fitting was applied to all available measured range data for data smoothing and variance calculation. Since a whole block of measured data are needed for the process of polynomial fitting, accurate and real-time mobile positioning may not be possible due to the time delay in collecting enough data. In other mobile location estimation methods, biased versions of the Kalman filter were used in mitigating the NLOS range error (Le et al., 2003). A coefficient for adjusting the noise covariance matrix needs to be chosen by experiment to obtain good location estimation results. In (Najar \& Vidal, 2003), a modified Kalman algorithm with NLOS error estimation was proposed for UMTS mobile positioning. The estimation of range bias in the algorithm provided performance improvement of location tracking in the NLOS environments.

Since most wireless communications systems used in wireless location may suffer from the NLOS and dense multipath situation, it is an important issue to obtain higher accuracy in determining signal arrival time for the time-based location systems. The ultra-wideband (UWB) radio system, in addition to the usage for communications, can provide users with the abilities of high accurate location estimation and tracking. As a good candidate for lowpower high-speed wireless communications, the ultra-wideband (UWB) radio technology has gained many interests in recent years for its applications in indoor communications. In indoor environments, the UWB systems, based on the spectral characteristics of the signals, are capable of tackling the multipath effects and providing finer and more accurate measurements in ranging than other narrow-band systems. With the fine time resolution, the accuracy of UWB location systems can be within one inch. The UWB systems though provide potentially accurate ranging for indoor positioning and tracking, the NLOS propagation errors caused by blocked LOS paths between a base station and the mobile 
station may still lead to severe degradation of position accuracy, posing a major challenge to positioning and tracking. Suitable NLOS identification and mitigation techniques are basically required in the systems for achieving better positioning performance.

This chapter will first present the UWB signal models in LOS and NLOS indoor environments. The data smoothing schemes using Kalman filter and polynomial fitting technique for identifying the NLOS status of the transmission are discussed. To improve the accuracy of time-based UWB range estimation, the NLOS mitigation techniques using biased Kalman filters will be covered. Some problems of applying the biased Kalman filtering in the NLOS identification and mitigation are addressed, followed by solutions to improving the correctness of hypothesis tests in the identification stage, and reducing the exceeding negative adjustment effects of biased Kalman filtering in the mitigation stage. The applications of extended Kalman filters on wireless positioning and tracking are then presented. A network-based location system, in which location metrics from multiple base stations are used for mobile target location estimation is studied. Positioning techniques using hybrid TDOA/AOA (time difference of arrival/angle of arrival) location metrics in the UWB environments will be presented. Simulation results are included to show the capability of Kalman filter-based architecture in mitigating the NLOS errors and improving the accuracy of target positioning and tracking in the UWB indoor wireless location system.

\section{Non-line of sight propagation errors}

\subsection{Range measurement model}

We assume that there are multiple base stations (BS's) in UWB indoor wireless location systems. In dealing with the non-line of sight propagation effects, the range measurement between a rover (or mobile station) and the $m$-th base station, corresponding to the TOA location metrics of the $m$-th base station can be modelled as

$$
r_{m}\left(t_{i}\right)=L_{m}\left(t_{i}\right)+n_{m}\left(t_{i}\right)+N \operatorname{LOS}_{m}\left(t_{i}\right)
$$

where $r_{m}\left(t_{i}\right)$ is the measured range at the sampling time $t_{i}, L_{m}\left(t_{i}\right)$ is the true range, $n_{m}\left(t_{i}\right)$ is the measurement noise and modelled as a zero-mean additive Gaussian random variable with standard deviation $\sigma_{m}$, and $\operatorname{NLOS}_{m}\left(t_{i}\right)$ is the NLOS error component in the received signal. There will be no NLOS error component if the line-of sight propagation path exists, and $\operatorname{NLOS}_{m}\left(t_{i}\right)=0$. The measurement error $n_{m}\left(t_{i}\right)$ becomes the only source of range measured error.

In a dense multipath UWB indoor environment, the estimation of the arrival time of the first path can be directly related to the measured range data at each base station, as in (1). The IEEE UWB channel modelling subcommittee adopted a modified Saleh-Velenzuela (S-V) model, which seemed to best fit the UWB channel measurements (Molisch, et al., 2003). The $\mathrm{S}-\mathrm{V}$ model was used in modelling the multipath of an indoor environment for wideband channel. The channel measurements showed that multipath arrivals in clusters rather than in a continuous form (Saleh \& Valenzuela, 1987), as shown in Fig. 1. Assume that $T_{0}$ is the arrival time of the first path in the first cluster. The arrival time $T_{0}$ can be related to the positive NLOS error component $\operatorname{NLOS}_{m}\left(t_{i}\right)$ at the time instant $t_{i}$. For the LOS cases, we have $T_{0}=0$ and $\operatorname{NLOS}_{m}(t)=T_{0} \times c=0$, where $c$ is the speed of light. 
The arrival time $T_{0}$ for the NLOS cases can be modelled as an exponential distribution and described by the following equation (Molisch, et al., 2003)

$$
p\left(T_{0}\right)=\Lambda \exp \left[-\Lambda\left(T_{0}\right)\right]
$$

where $\Lambda[1 / \mathrm{nsec}]$ is the cluster arrival rate. The multipath cluster arrival rates under different UWB channel models, CM1 through CM4, are listed in Table 1. The related parameters listed by the IEEE UWB channel modelling subcommittee have been used in many simulations and technical designs of a variety of UWB systems.

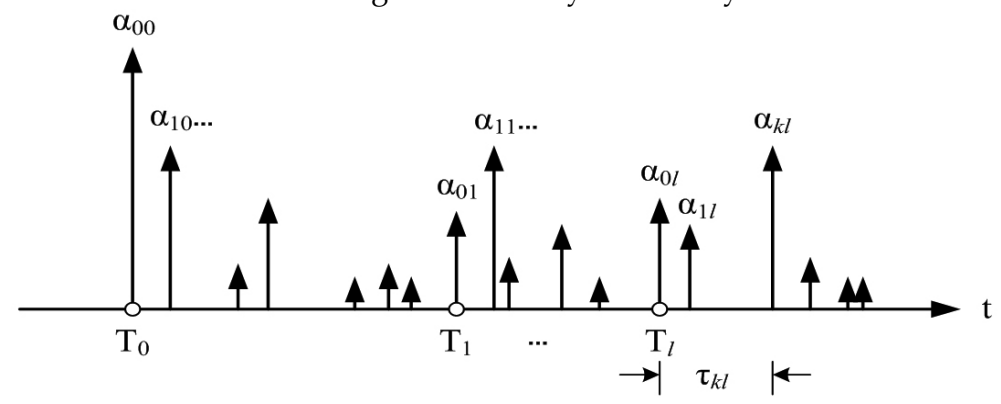

Fig. 1. Multipath delay profile of typical UWB channels

\begin{tabular}{|c|c|c|c|c|}
\hline UWB Channel & CM 1 & CM 2 & CM 3 & CM 4 \\
\hline Tx/Rx separation & $0-4 \mathrm{~m}$ & $0-4 \mathrm{~m}$ & $4-10 \mathrm{~m}$ & $>10 \mathrm{~m}$ \\
\hline$\Lambda(1 / \mathrm{nsec})$ & 0.0233 & 0.4 & 0.0667 & 0.0667 \\
\hline LOS/NLOS condition & LOS & NLOS & NLOS & NLOS \\
\hline
\end{tabular}

Table 1. The cluster arrival rate of multipath used in UWB channel models

\subsection{Non-line of sight identification}

For the NLOS error problems in mobile position location, several NLOS identification and mitigation techniques have been presented in the past few years (Thomas et al., 2000; Wylie \& Holtzmann, 1996). These approaches identified the BS's that have NLOS components in the received range data, and tried to reduce the time-based NLOS errors by using the NLOS mitigation techniques. In (Wylie \& Holtzmann, 1996), a simple binary hypothesis test was used for the NLOS identification with an understanding that the standard deviation of the NLOS propagation errors is generally much larger than that of measurement errors in the LOS situation. The understanding may also be applied to the UWB transmission environments. In (Wylie \& Holtzmann, 1996), prior to the binary hypothesis testing, polynomial fitting was applied to all available measured mobile range data collected during a block of time interval for variance calculation and data smoothing. Since the whole block of measured data were needed for the process of polynomial fitting, real-time positioning may not be feasible. For mitigation of the NLOS errors $\operatorname{NLOS}_{m}\left(t_{i}\right)$, the existence of non-zero NLOS component may need to be identified first. As shown in Fig. 2, the measured range data are first processed to obtain the smoothed data, which can further be used as averaged values at the corresponding time instants. The standard deviation of the measured range 
data with respect to the smoothed values can then be calculated and used in the proceeding LOS/NLOS hypothesis test.

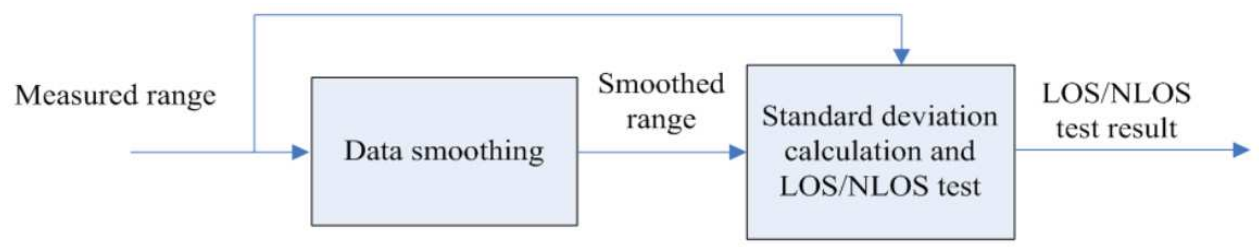

Fig. 2. Identification of LOS/NLOS propagation status

By applying the abovementioned identification scheme to wireless location systems, the range (or TOA) measurements related to each base station can be smoothed by using the least squares technique in solving the coefficients for fitting the modelled $N$-th order polynomial function (Wylie \& Holtzmann, 1996). The standard deviation of the measured range data can be obtained by computation over a block of $K$ range data $r_{m}\left(t_{i}\right)$ periodically:

$$
\hat{\sigma}_{m}=\sqrt{\frac{1}{K} \sum_{i=1}^{K}\left(r_{m}\left(t_{i}\right)-S_{m}\left(t_{i}\right)\right)^{2}}
$$

where $S_{m}\left(t_{i}\right)$ is the smoothed range data, which are obtained from polynomial fitting.

The data smoothing can also be conducted by other methods. In (Le et al., 2003), the range data were smoothed by utilizing biased Kalman filtering approach. In the case, the smoothed range data $S_{m}\left(t_{i}\right)$ were obtained from the output of the biased Kalman filter. The estimated standard deviation obtained in (3) is then compared with a predetermined threshold in the simple hypothesis testing, represented as

$$
\begin{array}{ll}
H_{0}: \hat{\sigma}_{m}(k)<\gamma \sigma_{m} & \text { LOS case } \\
H_{1}: \hat{\sigma}_{m}(k) \geq \gamma \sigma_{m} & \text { NLOS case }
\end{array}
$$

where $\sigma_{m}$ is the standard deviation of the measurement noise in the LOS environment. The scaling parameter $\gamma$ is chosen experimentally to reduce the probability of false alarm.

For the hypothesis testing in (Le et al., 2003), a periodical interval checking method was used. Periodical LOS/NLOS checking schemes, however, have some drawbacks. First of all, the block size of data samples for variance calculation needs to be chosen experimentally. Secondly, the period of using the hypothesis testing result may not be easily determined and must be decided experimentally as well. In Fig. 3, the block of $C$ data samples, shown as shaded bars $[0, C]$, are used for status checking. The resulting LOS/NLOS status will be kept unchanged until the time instant $N$, when another cycle of status identification begins and the new LOS/NLOS status are used. To choose feasible time instants $C$ and $N$ would need consideration of more factors in the transmission channels for the positioning systems. In addition to the problems, since the result of the LOS/NLOS hypothesis testing is used as the channel status until the next new periodical checking result is obtained, it is very likely that an NLOS-to-LOS or LOS-to-NLOS transition time instant is missed or incorrectly detected. The incorrect identification of channel status or the incorrect transition time may yield large estimation errors in ranging and localization. 


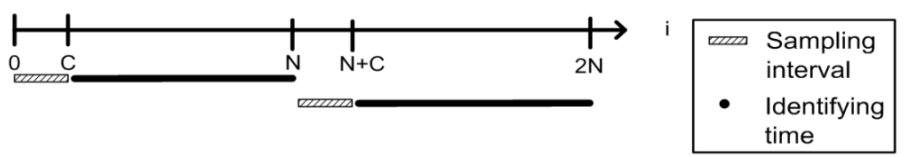

Fig. 3. Periodical interval checking for identifying LOS/NLOS status

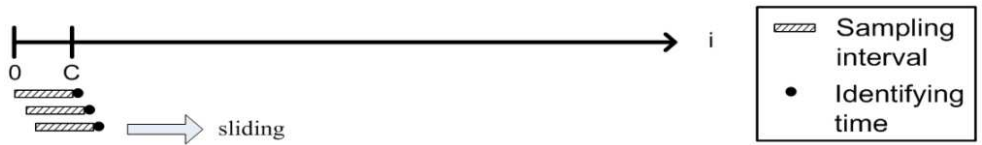

Fig. 4. Periodical interval checking for identifying LOS/NLOS status

To avoid the drawbacks, an identification scheme using a sliding window can be used in processing the measured range data at the base stations. The function of the sliding window spans from the data smoothing step, the calculation of standard deviation step to the LOS/NLOS identification step. A fixed-length data window of $C$ data samples, shown as the shaded bars $[0, C]$ in Fig. 4, will slide to the right in the time axis as the new data sample is available for processing. The sliding window scheme is considered compatible with the schemes using Kalman filtering.

\section{Kalman filtering for data processing}

\subsection{Data smoothing for the NLOS hypothesis testing}

A Kalman filter can be used in estimating the state vector of a mobile target from the observed range data, and therefore smoothing the range data. Assume that the state vector of a mobile can be represented as (Mendel, 1987)

$$
\mathbf{X}(k+1)=\mathbf{\Phi} \mathbf{X}(k)+\boldsymbol{\Gamma} W(k)
$$

where $\mathbf{X}(k)=\left[\begin{array}{lll}L(k) & \dot{L}(k) & \ddot{L}(k)\end{array}\right]^{T}$ is the state vector of the mobile target related to the measured data of a base station at the time $t_{k}, W(k)$ is the driving noise vector with an assumed covariance $Q=\sigma_{w}^{2}$, and the state transition and noise transition matrices can be written as

$$
\begin{gathered}
\boldsymbol{\Phi}=\left[\begin{array}{ccc}
1 & \Delta t & \Delta t^{2} / 2 \\
0 & 1 & \Delta t \\
0 & 0 & 1
\end{array}\right], \text { and } \\
\Gamma=\left[\begin{array}{c}
0 \\
0 \\
\Delta t
\end{array}\right] .
\end{gathered}
$$

The measurement process is represented as

$$
Z(k)=\mathbf{H X}(k)+U(k)
$$


where $Z(k)$ is the measured data, measurement matrix $\mathbf{H}=\left[\begin{array}{lll}1 & 0 & 0\end{array}\right]$, and $U(k)$ is the measurement noise with covariance $R=\sigma_{u}^{2}$. The iterative operations of the Kalman filter can be summarized as follows:

$$
\begin{gathered}
\widehat{\mathbf{X}}(k \mid k-1)=\mathbf{\Phi} \widehat{\mathbf{X}}(k-1 \mid k-1) \\
\mathbf{P}(k \mid k-1)=\mathbf{\Phi} \mathbf{P}(k-1 \mid k-1) \mathbf{\Phi}^{T}+\mathbf{\Gamma} \mathbf{Q} \boldsymbol{\Gamma}^{T} \\
\mathbf{K}(k)=\mathbf{P}(k \mid k-1) \mathbf{H}^{T}\left[\mathbf{H P}(k \mid k-1) \mathbf{H}^{T}+\mathbf{R}(k)\right]^{-1} \\
\widehat{\mathbf{X}}(k \mid k)=\widehat{\mathbf{X}}(k \mid k-1)+\mathbf{K}(k)[Z(k)-\mathbf{H} \widehat{\mathbf{X}}(k \mid k-1)] \\
\mathbf{P}(k \mid k)=\mathbf{P}(k \mid k-1)-\mathbf{K}(k) \mathbf{H P}(k \mid k-1)
\end{gathered}
$$

where $\mathbf{K}(k)$ is the Kalman gain vector and $\boldsymbol{P}(k \mid k)$ is the covariance matrix of $\widehat{\mathbf{X}}(k \mid k)$.

By using the Kalman filtering, the standard deviation of the observed range data can be calculated and then used in the LOS/NLOS hypothesis testing. To avoid the drawbacks of using polynomial fitting and periodical interval checking methods, as discussed in the previous subsection, the sliding window scheme for processing the measured range data can be integrated with the biased Kalman filtering and hypothesis testing in the UWB location system. The standard deviation of data over a sliding block of $C$ TOA (range) measurements $r_{m}\left(t_{i}\right)$ at the $m$-th base station can be obtained as

$$
\hat{\sigma}_{m}(k)=\sqrt{\frac{1}{N} \sum_{i=k-(C-1)}^{k}\left(r_{m}\left(t_{i}\right)-\hat{L}_{m}\left(t_{i}\right)\right)^{2}} .
$$

In each processing cycle, the standard deviation calculated at base station $m$ is then used in the hypothesis testing, as represented in (4). If the LOS TOA propagation status is decided, an unbiased Kalman filter is used to smooth the TOA data at each BS. In the contrast, if the NLOS propagation scenario is detected, a biased Kalman filter is used in mitigating the NLOS TOA error.

\subsection{Biased Kalman filtering}

In a biased Kalman filter, based on the result from LOS/NLOS hypothesis testing, different values of noise covariance are assigned. Under the LOS situation, unbiased smoothing is used for estimating the true TOA. When the NLOS status is detected, the positive NLOS range error in the measured range (or TOA) data can be effectively reduced in the filtering cycle by assigning the diagonal elements of the noise covariance matrix

$$
\begin{aligned}
& \hat{\sigma}_{u}(k)=\alpha \sigma_{m}, \text { if } Z(k)-\mathbf{H X}(k \mid k-1)>0 \text { and NLOS detected, } \\
& \quad=\sigma_{m}, \quad \text { otherwise, }
\end{aligned}
$$

where $\alpha$ is an experimentally chosen scaling factor. The processed TOA data from all base stations are then used in mobile localization and tracking. 


\subsection{Biased Kalman filtering for TDOA location metrics}

Similar to the cases in the TOA location methods, the Biased Kalman filters can also be applied in the TDOA location approaches. Assume that there are $M$ base stations available for measuring the TOA's for locating a mobile station. The first base station, BS1 is selected as the reference base station. The difference of ranges of the $m$-th base station and the first base station to the mobile station in the location system can be modelled as

$$
\begin{aligned}
d_{m}\left(t_{i}\right) & =r_{m}\left(t_{i}\right)-r_{1}\left(t_{i}\right) \\
& =L_{m}\left(t_{i}\right)-L_{1}\left(t_{i}\right)+n_{d, m}\left(t_{i}\right)+N \operatorname{LOS}_{d, m}\left(t_{i}\right) \\
m & =2, \ldots, M
\end{aligned}
$$

where $n_{d, m}\left(t_{i}\right)$ is defined as the measurement noise of the range difference, $n_{d, m}\left(t_{i}\right)=n_{m}\left(t_{i}\right)-n_{1}\left(t_{i}\right)$, and $\operatorname{NLOS}_{d, m}\left(t_{i}\right)$ is the NLOS error component of the range difference, $\operatorname{NLOS}_{d, m}\left(t_{i}\right)=\operatorname{NLOS}_{m}\left(t_{i}\right)-\operatorname{NLOS}_{1}\left(t_{i}\right)$. Hence, $n_{d, m}\left(t_{i}\right)$ can be modelled as a random variable, at time instant $t_{i}$, with independently identical joint probability density function with $n_{d, m}\left(t_{i}\right) \sim N\left(0,2 \sigma_{m}^{2}\right)$.

To perform range difference estimation in the TDOA data processing, the Kalman filtering scheme discussed above can be applied in the cases here. Three cases with different LOS/NLOS combinations are considered for determining the biased measurement noise covariance $R=\hat{\sigma}_{u}^{2}$ in the system.

Case 1: BS $m$ is an NLOS BS, and BS 1 is an LOS BS. We have

$$
Z_{m}(k+1)-\mathbf{H} \mathbf{X}_{m}(k+1 \mid k)>0
$$

Case 2: BS 1 is an NLOS BS, and BS $m$ is an LOS BS. We have

$$
Z_{m}(k+1)-\mathbf{H} \mathbf{X}_{m}(k+1 \mid k)<0
$$

Case 3: Both BS $m$ and BS 1 are NLOS BS's.

When the three different cases are not met, both BS $m$ and BS 1 are considered LOS BS's. From the results of case determination, the value of $\hat{\sigma}_{u}^{2}$ can be assigned by using the following rules:

$$
\hat{\sigma}_{u}= \begin{cases}\alpha \sigma_{m}, & \text { Case } 1 \text { or Case 2, } \\ \sqrt{2} \frac{c}{\Lambda} \sigma_{m}, & \text { Case } 3, \\ \sqrt{2} \sigma_{m}, & \text { otherwise, }\end{cases}
$$

where $\alpha$ is experimentally chosen scaling factors, $c$ is the speed of light, $\Lambda$ is the cluster arrival rate and $\sigma_{m}$ is the standard deviation of AWGN measurement noise. If an all-LOS scenario exists, the range difference estimation is constructed by an unbiased Kalman filter; otherwise, the range difference is estimated by using a biased Kalman filter.

\section{Range estimation with modified biased Kalman filtering}

\subsection{Problems in NLOS identification using Kalman filter and sliding window}

In data smoothing, the combination of Kalman filtering and sliding window scheme has been considered a possible solution to the shortcomings caused by using polynomial fitting 
or periodical interval checking. Problems remain when trying to identify the NLOS-to-LOS channel status transition. In Fig. 5, it can be seen that the range data smoothed by the Kalman filter decrease slower than expected. The phenomenon may cause a time period with identification errors in the proceeding hypothesis test. During the falling edge of transition, earlier values of the smoothed range data may yield standard deviations which will be larger than the threshold in the hypothesis test, as represented in (4). Instead of using the outputs of Kalman filter as the smoothed range data, a modified scheme is considered for directly generating range estimates. The formulation will be discussed in Section 4.3.

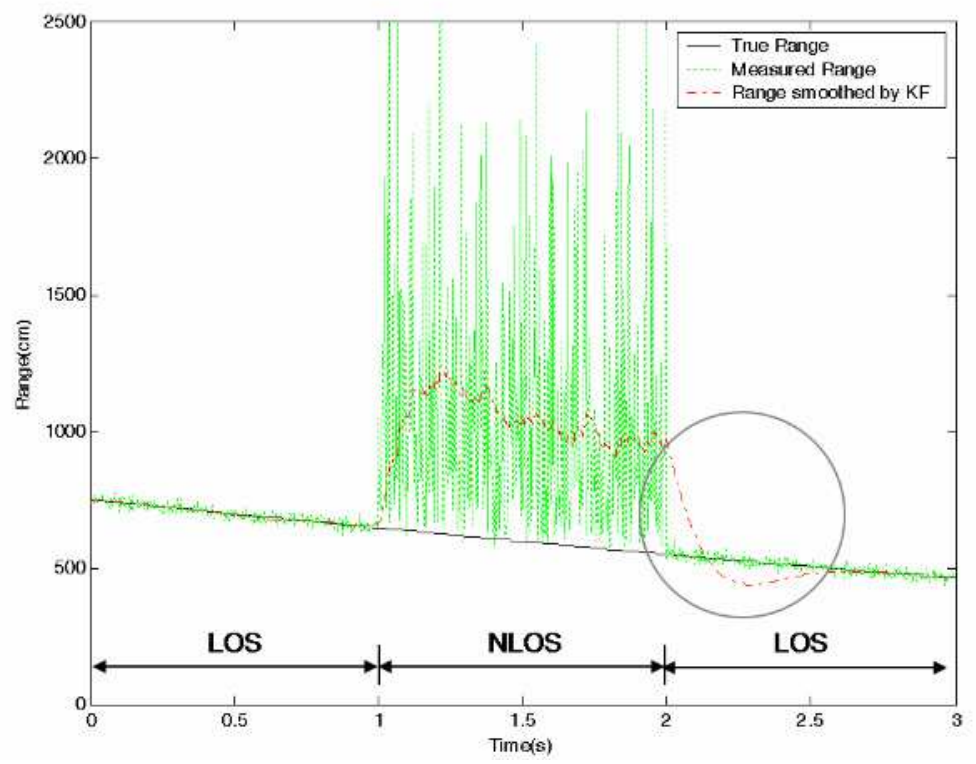

Fig. 5. Slow falling transition of range data smoothed by Kalman filter during the NLOS-toLOS change of channel status

\subsection{Problem in NLOS mitigation using biased Kalman filter}

The bias adjusting rule in (15) was used in the hope that the positive NLOS propagation errors be mitigated effectively by increasing the estimated noise standard deviation. The positive NLOS range bias can be reduced by assigning the diagonal elements of noise covariance matrix. However, a resultant side effect is that exceeding negative adjustments may occur in the beginning part of the NLOS status, as shown in Fig. 6. Generally speaking, if an NLOS status is detected and the innovation from the measured data is positive, the measured data may be treated as affected by noise with larger variance and the adjusting rule will achieve the objective of NLOS mitigation in the iteration. In the contrast, if the innovation from the measured data is negative, a possible LOS status would be assumed. The assumption may become incorrect in the next iteration, when the channel is actually in NLOS status. The error would yield an even more negative adjustment in the estimated range value. To tackle the undesirable effect, a new bias adjusting rule is required. 


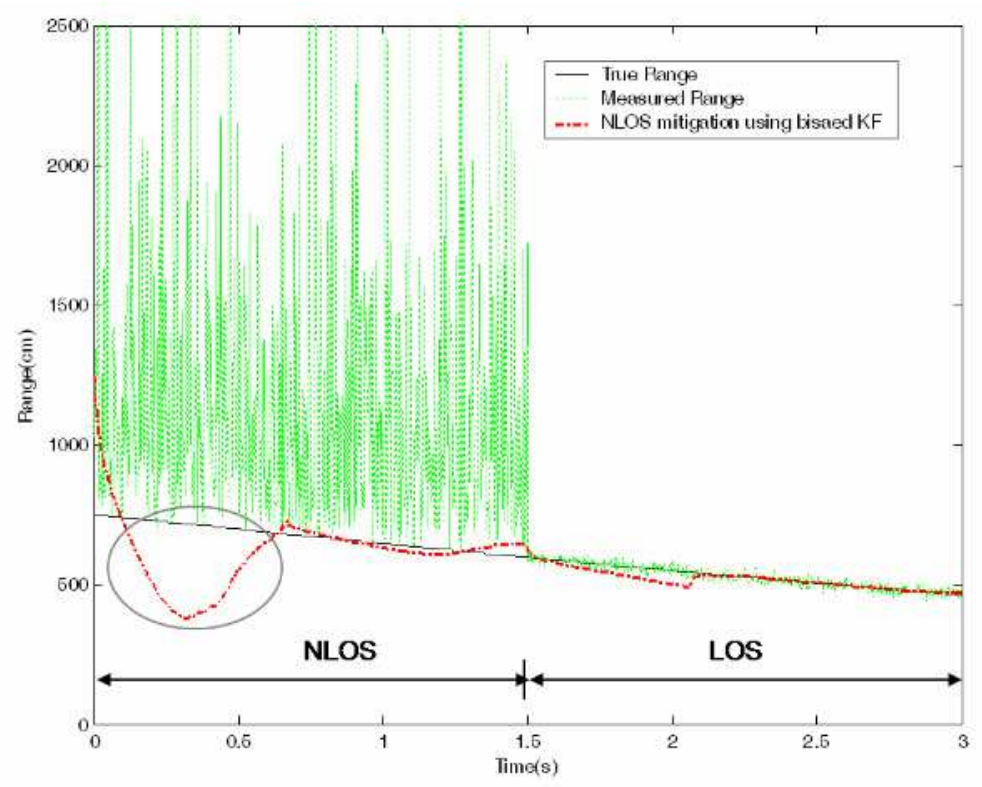

Fig. 6. Exceeding negative adjustments in the beginning part of the NLOS status,

\subsection{Functional combination of NLOS identification and mitigation}

A novel range estimation scheme with a modified biased Kalman filter for NLOS range mitigation and LOS/NLOS identification is presented and shown in Fig. 7. The modified biased Kalman filter is used to process the range (or TOA) measurement according to the feedback identification result from the processed data in the previous iteration of Kalman filtering. Before computing the Kalman gain in (11), the measurement noise covariance $\sigma_{u}^{2}$ or the range prediction covariance $P_{1,1}(k \mid k-1)$ must be adjusted by the following adjusting rules.

For NLOS case and $Z(k)-\mathbf{H X}(k \mid k-1)>0$, let

$$
\hat{\sigma}_{u}^{2}=\hat{\sigma}_{m}^{2}(k-1)+\text { function(innovation); }
$$

For NLOS case and $Z(k)-\mathbf{H X}(k \mid k-1)<0$, let

$$
\begin{gathered}
\hat{\sigma}_{u}^{2}(k)=\sigma_{m}^{2} \text {, and } \\
P_{1,1}(k \mid k-1)=P_{1,1}(k \mid k-1)+\text { function(innovation) } ;
\end{gathered}
$$

For LOS case, let

$$
\hat{\sigma}_{u}^{2}(k)=\sigma_{m}^{2}
$$

where $\hat{\sigma}_{m}^{2}(k-1)$ is the standard deviation obtained by the sliding window in the previous iteration. By adjusting noise covariance in (18) and (19), the positive NLOS range error can 
be significantly reduced. The inclusion of (20) is essential in compensating the range prediction covariance $P_{1,1}(k \mid k-1)$. The biased term avoids inaccurate estimation of the range rate $\dot{L}_{m}(k)$ from the NLOS mitigation.

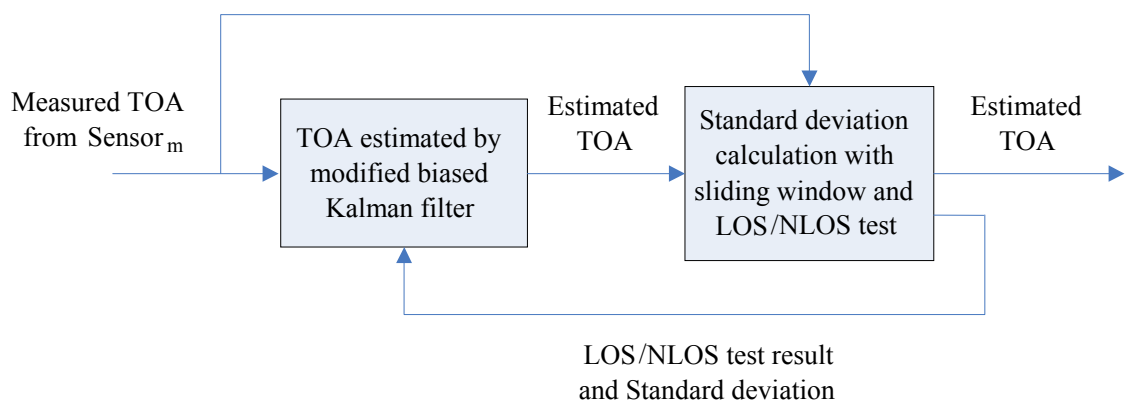

Fig. 7. Range (TOA) mitigation and LOS/NLOS identification

In the range estimation scheme, measured TOA data are first processed by a modified biased Kalman filter. Based on the LOS/NLOS status and the standard deviation feedback from the previous processed data, the modified biased Kalman filter generates an estimated TOA for the current processed data. Under the NLOS situation, the measured TOA's are smoothed by using biased filters, and the positive NLOS errors are mitigated. The standard deviation from the feedback path is regarded as a reference representing the degree of NLOS errors. Under the LOS situation, unbiased smoothing is used for estimating the true TOA value.

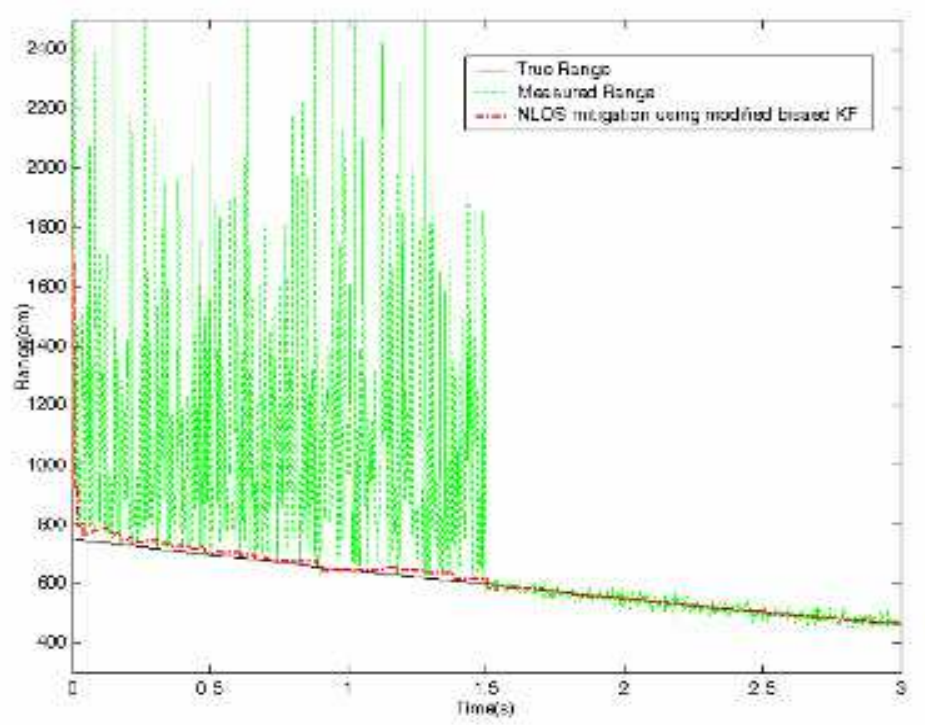

Fig. 8. Results of using the range estimation scheme with a modified biased Kalman filter 
In the second functional block in Fig. 7, the standard deviation of the estimated TOA is calculated. With a sliding window, the standard deviation of the last $C$ estimated TOA's is calculated. The obtained standard deviation is passed through an LOS/NLOS hypothesis testing to determine the status of propagation. The resultant LOS/NLOS status and the standard deviation are then used as feedback to the modified biased Kalman filter for data processing in the next cycle. The design objective is that the transition between LOS and NLOS conditions can be detected immediately and the NLOS effects can be effectively mitigated in order to obtain a sequence of estimated TOA's, which are close to the corresponding true range between the mobile and a base station. Fig. 8 shows the results of applying the novel range estimation scheme with a modified biased Kalman filter for NLOS range mitigation and LOS/NLOS identification

For a location system with multiple base stations, the processed TOA data from all participating base stations are then used in positioning and tracking. Formulated TDOA data are also possible if TDOA location system is desired.

\section{Hybrid TDOA/AOA indoor positioning and tracking}

\subsection{Extended Kalman filter for TDOA/AOA positioning}

Both time-based and angle-based categories have their own advantages and limitations, it is therefore reasonable to consider hybrid methods to integrate the merits of using the two types of metrics. In (Cong \& Zhuang, 2001), a hybrid TDOA/AOA location scheme was presented for wideband code division multiple access (WCDMA) systems. The scheme uses TDOA information from all base stations (BS's) and the AOA information at the serving base station to perform mobile location estimation.

In other mobile location methods, biased versions of the Kalman filter were used in mitigating the NLOS range error. With the rule-determined coefficient for the measurement noise covariance matrix, good location estimation results would be obtained (Le et al., 2003; Thomas et al., 2000). In (Najar \& Vidal, 2003), Kalman filtering algorithm with NLOS bias estimation was proposed for UMTS mobile positioning. The estimation of range bias provided performance improvement of location tracking in NLOS environments.

To meet the demand of high location accuracy in indoor positioning applications, the UWB system is considered due to the fine time resolution. The fine resolution of UWB signals provides potentially accurate ranging for indoor location communications, where dense multipath and NLOS errors are the major challenge to the quality of indoor positioning applications. To improve the accuracy of positioning, methods for eliminating or mitigating the effects of NLOS errors and multipath in the UWB environments need to be applied before the TDOA/AOA location technique is used.

For designing suitable NLOS identification and mitigation algorithms for the UWB systems, parameters of the standard UWB channel models provided by the IEEE 802.15.3a standards task group are used in the studies and simulations. In contrast to the scheme in (Cong \& Zhuang, 2001), all "good" AOA data along with TDOA information from all BA's are considered in locating the MS position. The AOA and TDOA information are processed centrally by the extended Kalman filter (EKF) for MS positioning and tracking. The architecture of location estimator is illustrated in Fig. 9. For attaining effective NLOS mitigation and obtaining more accurate TOA estimates, the functional blocks in each branch may be replace by the scheme shown in Fig. 7 . 


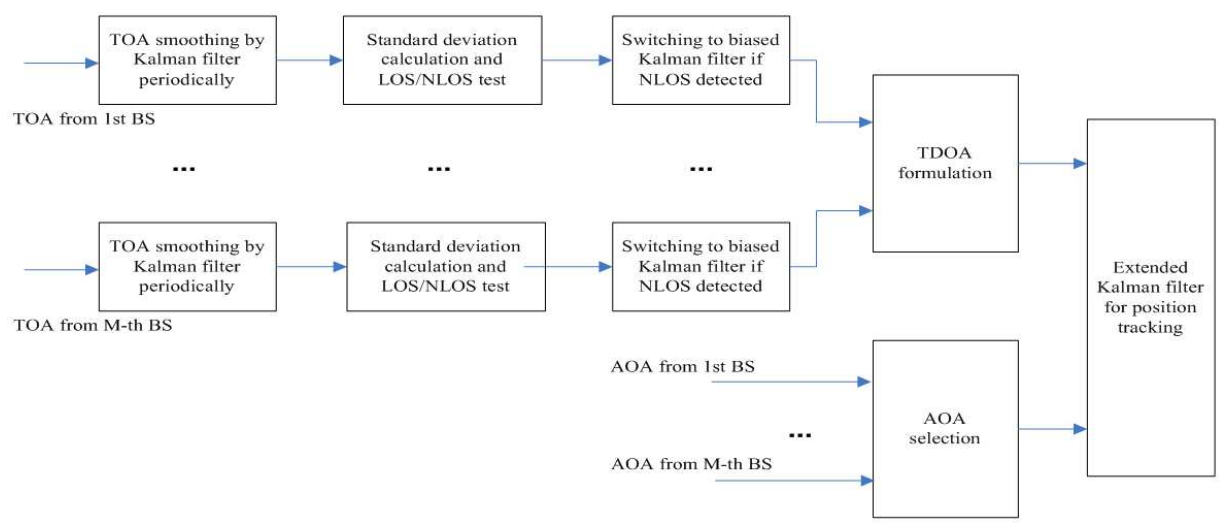

Fig. 9. Hybrid TDOA/AOA positioning and tracking

In Fig. 9, the NLOS error mitigation consists of two parts: the NLOS TOA error mitigation and the AOA information selection. If the LOS TOA propagation scenario is decided, an unbiased Kalman filter is used to smooth the TOA data at each BS. In the contrast, if the NLOS propagation scenario is detected, a biased Kalman filter or modified biased Kalman filter is used in mitigating the NLOS TOA errors. The positive NLOS range bias can be reduced by assigning the diagonal elements of noise covariance matrix. The processed TOA data from all base stations are then used in formulating the TDOA data, which can be further used for mobile positioning and tracking.

The AOA information from all base stations are processed by the AOA selection to avoid introducing large NLOS bearing error into the position tracking stage. Only AOA data from LOS base stations are selected for further processing. In other words, any NLOS AOA data will be discarded.

The formulated TDOA data and the selected AOA data are processed by the extended Kalman filter for the MS location estimation. The state vector of a mobile station is defined as

$$
\mathbf{S}(k \mid k-1)=\boldsymbol{\Phi}^{\prime} \mathbf{S}(k-1 \mid k-1)+\mathbf{W}^{\prime}(k-1)
$$

where $\mathbf{s}(k)=\left[\begin{array}{llll}x(k) & y(k) & \dot{x}(k) & \dot{y}(k)\end{array}\right]^{T}$ is the state vector at time instant $k$. The covariance matrix of the driving noise vector $\mathbf{W}^{\prime}(k)$ is

and the state transition matrix is

$$
\mathbf{Q}^{\prime}=\left[\begin{array}{cccc}
0 & 0 & 0 & 0 \\
0 & 0 & 0 & 0 \\
0 & 0 & \sigma_{u}^{2} & 0 \\
0 & 0 & 0 & \sigma_{u}^{2}
\end{array}\right],
$$

$$
\boldsymbol{\Phi}^{\prime}=\left[\begin{array}{llll}
1 & 0 & T & 0 \\
0 & 1 & 0 & T \\
0 & 0 & 1 & 0 \\
0 & 0 & 0 & 1
\end{array}\right] .
$$


In the case where the LOS status exists between the mobile station and all base stations, the TDOA/AOA measurement process can be represented as

$$
\mathbf{Z}(k)=f(\mathbf{S}(k))+\mathbf{U}^{\prime}(k),
$$

where $\mathbf{Z}(k)$ is the measured data vector, $f(\mathbf{S}(k))$ is a nonlinear transformation, and $\mathbf{U}^{\prime}(k)$ is the measurement noise. The covariance matrix of $\mathbf{U}^{\prime}(k)$ is

$$
\mathbf{R}^{\prime}=\left[\begin{array}{cc}
\mathbf{H} \boldsymbol{\sigma}^{2} \mathbf{H}^{T} & \mathbf{0} \\
\mathbf{0} & \sigma_{\mathrm{AOA}}^{2} \mathbf{I}
\end{array}\right]_{(2 \mathrm{M}-1) \times(2 \mathrm{M}-1)},
$$

in which the TDOA formulation can be written as

$$
\mathbf{H}=\left[\begin{array}{ccccc}
-1 & 1 & 0 & \cdots & 0 \\
-1 & 0 & 1 & \cdots & 0 \\
\vdots & \vdots & \vdots & \ddots & \vdots \\
-1 & 0 & \cdots & 0 & 1
\end{array}\right]_{(\mathrm{M}-1) \times \mathrm{M}}
$$

and

$$
\boldsymbol{\sigma}^{2}=\left[\begin{array}{cccc}
\sigma_{R}^{2} & 0 & \cdots & 0 \\
0 & \sigma_{R}^{2} & \cdots & 0 \\
\vdots & \vdots & \ddots & \vdots \\
0 & 0 & \cdots & \sigma_{R}^{2}
\end{array}\right]_{\mathrm{M} \times \mathrm{M}},
$$

where $\sigma_{\mathrm{R}}^{2}$ is the variance of the range related to the output of the unbiased or biased Kalman filter. The AOA variance $\sigma_{\mathrm{AOA}}^{2}$ is related to the selected LOS AOA data. The dimension of the matrix $\mathbf{I}, m \times m$ is the determined number of LOS base stations from the AOA selection. As shown in Fig. 10, the results from the LOS/NLOS hypothesis are used in selecting the LOS AOA metrics.

When the NLOS situation occurs, the covariance matrices of the processed TDOA and AOA data are different from those in the LOS situation. The dimension of the matrix in measurement process for the NLOS situations will be decreased, and determined by the sum of the number of TDOA and the number of LOS AOA data.

\subsection{Simulations results and discussions}

The performance of the hybrid TDOA/AOA positioning technique for indoor UWB systems is studied by conducting computer simulations. We assume that three base stations are used in the location system. The coordinates are BS1: $(0,0)$, BS2: $(5 \mathrm{~m}, 8.66 \mathrm{~m})$, and BS3: $(10 \mathrm{~m}, 0)$, respectively. The NLOS range error is assumed to be an exponential distribution, which is defined in the standard indoor UWB channel model (Foerster, 2003; Molisch, et al., 2003). A multipath cluster arrival rate $0.0667^{*} 10^{9}$ for $\mathrm{CM} 3$ is used for the case where the distance 


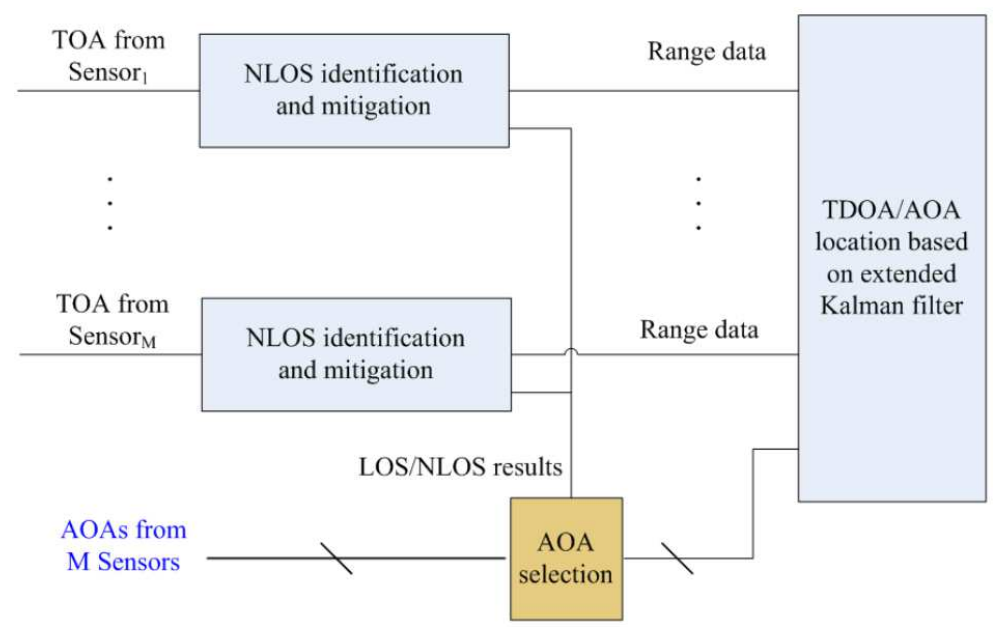

Fig. 10. Hybrid TDOA/AOA positioning and tracking with AOA selection

between any BS and the MS is within the range from 4 to 10 meters. The NLOS bearing is assumed to be uniformly distributed from $-\pi$ to $\pi$. It is assumed that an MS travels from location $(7 \mathrm{~m}, 4.5 \mathrm{~m})$ to $(4 \mathrm{~m}, 0.5 \mathrm{~m})$ with a constant velocity, $0.5 \mathrm{~m} / \mathrm{s}$, as illustrated in Fig. 11 . The observed time length is 10s, and the sample spacing is 25ms. Two LOS/NLOS propagation scenarios are investigated.

In the first scenario, the LOS propagation between the MS and BS3 turns NLOS at $t=2 s$, and remains NLOS until $t=10$ s. The simulation results in Fig. 12 shows that the hybrid TDOA/AOA positioning scheme with the AOA selection function performs well in terms of root mean square location errors. Without the AOA selection, the NLOS bearing error may lead to severe degradation of position accuracy.
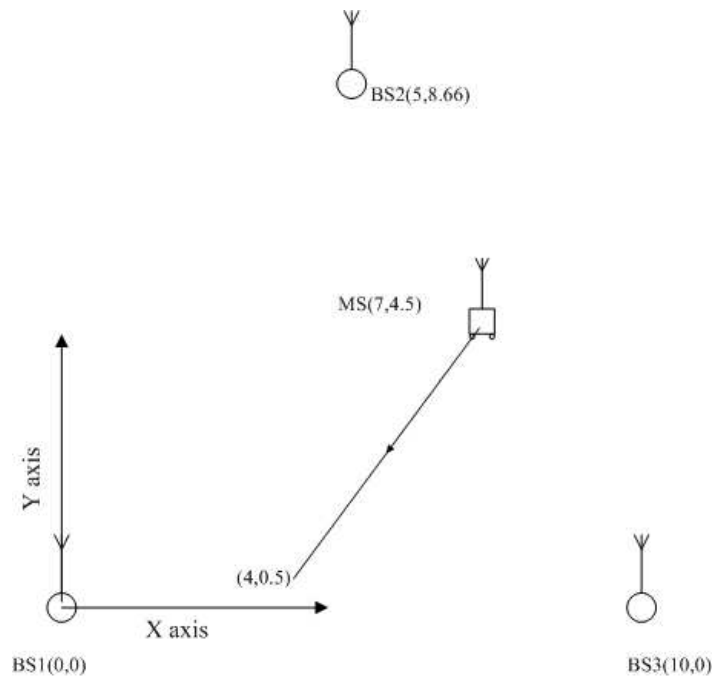

Fig. 11. A simulation example with three BS's and one mobile target 


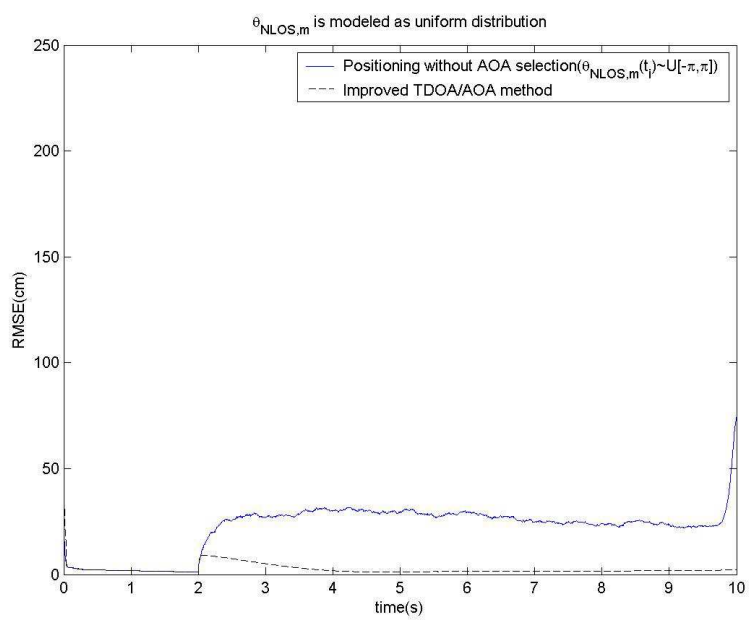

Fig. 12. Performance of positioning with and without AOA selection (Scenario 1: one NLOS BS is assumed)

In the second scenario, the propagation between the MS and two BS's (BS2 and BS3) turn NLOS at $t=2 s$, and remains NLOS until $t=10 \mathrm{~s}$. In the situation, the data available to the extended Kalman filter will change from three AOA's and two TDOA's to one selected AOA and two TDOAs. The simulation results are shown in Fig. 13. It can be seen that the position errors are a bit larger in this case. The increased errors are mainly due to the larger residual errors at the NLOS TOA mitigation stage and the processing of fewer available AOA data in the adjustable TDOA/AOA-based extended Kalman filter for positioning.

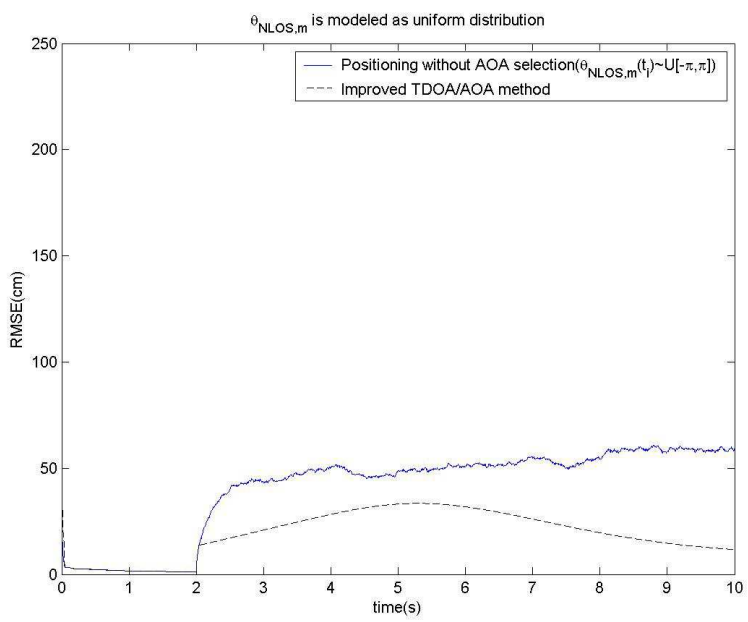

Fig. 13. Performance of positioning with and without AOA selection (Scenario 2: Two NLOS BS's are assumed) 


\section{Conclusion}

We present the applications of Kalman filter and extended Kalman filter on data smoothing, NLOS identification, NLOS mitigation and mobile target tracking in the UWB indoor wireless environments. To improve the accuracy of time-based UWB ranging, data smoothing with Kalman filtering for the NLOS hypothesis testing has been discussed. For the function of NLOS mitigation, biased Kalman filtering is investigated. To tackle the undesirable detection problems of using data smoothing and biased Kalman filtering in range estimation, a novel modified biased Kalman filtering scheme is presented. In the modified biased Kalman filtering scheme, functional combination of NLOS identification and NLOS mitigation is discussed. To investigate mobile target positioning and tracking in the NLOS indoor environments, with a focus on the network-based location system, where multiple base stations are involved in the location estimation, a hybrid TDOA/AOA location system formulated with extended Kalman filters is proposed. Simulation results show the Kalman filter-based scheme is capable of effectively mitigating the NLOS errors and therefore improving the accuracy of target positioning and tracking. Further efforts of NLOS mitigation will lead to improved estimation of signal arrival time and more accurate mobile target positioning and tracking. The integration of multiple Kalman filters and statistical data fusion schemes may also provide a promising solution to the emerging tracking applications using multiple mobile robots and wireless sensor networks in indoor wireless environments.

\section{References}

Cong, L. \& Zhuang W. (2001). Non-Line-of-Sight Error Mitigation in TDOA Mobile Location, Proceedings of IEEE Global Telecommunications Conference, Vol. 1, pp. 680684, San Antonio, TX. USA, Nov. 2001.

Foerster, J. Editor. (2003). Channel Modeling Sub-committee Report Final. Document IEEE P802.15-02/490r1-SG3a, IEEE.

Le B. L.; Ahmed K. \& Tsuji H. (2003). Mobile Location Estimator with NLOS Mitigation using Kalman Filtering. Proceedings of IEEE Wireless Communications and Networking Conference, Vol. 3, pp. 16-20, March 2003.

Mendel, J. M. (1987). Lessons in Digital Estimation Theory, Prentice-Hall, Inc., ISBN: 0-53406660-7, Upper Saddle River, NJ, USA.

Molisch, A. F.; Foerster, J. R. \& Pendergrass, M. (2003). Channel Models for Ultrawideband Personal Area Networks. IEEE Wireless Communications, Vol. 10, No. 6, December 2003, 14-21, ISSN: 1536-1284.

Najar M. \& Vidal J. (2003). Kalman Tracking for Mobile Location in NLOS Situations. Proceedings of The 14th IEEE International Symposium on Personal, Indoor and Mobile Radio Communication, Vol. 3, pp. 2203-2207, Sept. 2003.

Pahlavan, K.; Krishnamurthy P. \& Beneat, J. (1998) Wideband Radio Channel Modeling for Indoor Geolocation Applications. IEEE Communications Magazine, Vol. 36, No. 4, April 1998, 60-65.

Saleh, A. \& Valenzuela, R. (1987). A Statistical Model for Indoor Multipath Propagation. IEEE Journal on Selected Areas in Communications, Vol. SAC-5, No. 2, Feb. 1987, 128-137, ISSN: 0733-8716. 
Thomas, N. J.; Cruickshank, D. G. M. \& Laurenson, D. I. (2000). A Robust Location Estimator Architecture with Biased Kalman Filtering of TOA Data for Wireless Systems. Proceedings of IEEE 6th International Symposium on Spread-Spectrum Techniques and Applications, pp.296-300, Sept. 2000.

Wylie M. P. \& Holtzmann J. (1996). The Non-Line of Sight Problem in Mobile Location Estimation. Proceedings of IEEE International Conference on Universal Personal Communications, pp. 827-831, 1996. 


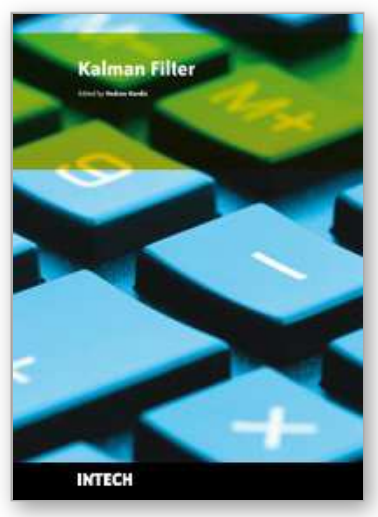

\author{
Kalman Filter \\ Edited by Vedran Kordic
}

ISBN 978-953-307-094-0

Hard cover, 390 pages

Publisher InTech

Published online 01, May, 2010

Published in print edition May, 2010

The Kalman filter has been successfully employed in diverse areas of study over the last 50 years and the chapters in this book review its recent applications. The editors hope the selected works will be useful to readers, contributing to future developments and improvements of this filtering technique. The aim of this book is to provide an overview of recent developments in Kalman filter theory and their applications in engineering and science. The book is divided into 20 chapters corresponding to recent advances in the filed.

\title{
How to reference
}

In order to correctly reference this scholarly work, feel free to copy and paste the following:

Chin-Der Wann (2010). Kalman Filtering for NLOS Mitigation and Target Tracking in Indoor Wireless Environment, Kalman Filter, Vedran Kordic (Ed.), ISBN: 978-953-307-094-0, InTech, Available from: http://www.intechopen.com/books/kalman-filter/kalman-filtering-for-nlos-mitigation-and-target-tracking-inindoor-wireless-environment

\section{INTECH}

open science | open minds

\author{
InTech Europe \\ University Campus STeP Ri \\ Slavka Krautzeka 83/A \\ 51000 Rijeka, Croatia \\ Phone: +385 (51) 770447 \\ Fax: +385 (51) 686166 \\ www.intechopen.com
}

\author{
InTech China \\ Unit 405, Office Block, Hotel Equatorial Shanghai \\ No.65, Yan An Road (West), Shanghai, 200040, China \\ 中国上海市延安西路65号上海国际贵都大饭店办公楼405单元 \\ Phone: +86-21-62489820 \\ Fax: +86-21-62489821
}


(C) 2010 The Author(s). Licensee IntechOpen. This chapter is distributed under the terms of the Creative Commons Attribution-NonCommercialShareAlike-3.0 License, which permits use, distribution and reproduction for non-commercial purposes, provided the original is properly cited and derivative works building on this content are distributed under the same license. 